\title{
Tuning of Thermo-Mechanical Performance: Modified Multiwalled Carbon Nanotubes Reinforced SBR/NBR/SR Nanocomposites
}

\author{
SADIA Sagar Iqbal ${ }^{1, a,{ }^{*}}$, MUHAMMAD Adrees $^{1 \mathrm{~b}}$, ADNAN Ahmad $^{1 \mathrm{c}}$, \\ FAIZA Hassan ${ }^{2 \mathrm{~d}}$, MUHAMMAD Yasir ${ }^{3 \mathrm{e}}$, ARSHAD Bashir ${ }^{4 \mathrm{f}}$, \\ SAJID Rashid Ahmad ${ }^{5 \mathrm{~g}}$, FAHAD Jamshad ${ }^{\text {ih }}$, WAHEED Gul Khan ${ }^{4 \mathrm{i}}$ \\ ${ }^{1}$ Department of Polymer Engineering \& Technology, CEET, \\ University of the Punjab, Lahore, Pakistan \\ ${ }^{2}$ Department of Chemistry, University of the Lahore, Pakistan \\ ${ }^{3}$ Institute of Space Technology, Islamabad, Pakistan \\ ${ }^{4}$ Institute of Industrial Control System, P.O. Box 1398, Rawalpindi, Pakistan \\ ${ }^{5}$ College of Earth and Environmental Sciences, University of the Punjab, Pakistan \\ ${ }^{*}$ asadia.pet.ceet@pu.edu.pk, ${ }^{b}$ muhammadadrees2002@gmail.com, \\ cadnanahmedbaloch@gmail.com, ${ }^{\mathrm{d}}$ faiza.hassan@chem.uol.edu.pk, \\ emuhammadyasir85@gmail.com, ${ }^{\mathrm{f}} \mathrm{hmarshid@yahoo.com,}{ }^{9}$ principal.cees@pu.edu.pk, \\ hfhdjamshed@gmail.com, 'jadoon_gul@yahoo.com
}

Keywords: Elastomeric composite, Functionalized multiwalled carbon nanotubes, Thermal transport, Thermal insulation, Thermal decomposition, Endothermic capability.

\begin{abstract}
The present study aimed to identify the potential of modified nano-reinforcement (multiwalled carbon nanotubes; m-MWCNTs) to attenuate the thermal transport/ decomposition/ transition and mechanical aspects of three different polymeric matrices. In order to develop strong interfacial interaction between the host matrix and the incorporated nanotubes, 3aminopropyletrimethoxy silane (APTMS) was used to m-MWCNTs. IR spectra confirmed the silane chemical moiety attachment on the upper surface of the MWCNTs. Conventional elastomeric mixing techniques were adopted to disperse m-MWCNTs within the three polymeric matrices (Acrylonitrile butadiene rubber, Silicone rubber, and Styrene Butadiene rubber) separately. SEM images assured the uniform dispersion of m-MWCNTs within the host polymeric matrices. Experimental evaluation of thermal conductivity revealed the reduction of thermal transport through the developed composite specimens by increasing the host polymer matrix to nano-filler concentration (m-MWCNTs). The utmost insulation effect was perceived in the F-MWCNTs incorporated silicone rubber nanocomposite comparatively. Glass transition/crystallization temperatures of the nanocomposites were lessened however melting temperatures were enhanced by impregnating nanotubes into the host polymeric matrices. Maximum thermal stability improvement due to the addition of m-MWCNTs was observed in the silicone elastomeric nanocomposite as compared to the other two systems. Proper dispersion and compatibility of m-MWCNTs with the polymeric matrices effectively enhanced the ultimate tensile strength (UTS)/elongation at break along hardness of rubber of the nanocomposites. The insulation character of $\mathrm{m}-\mathrm{MWCNTs} / \mathrm{silicone}$ rubber system was found best among the explored nanocomposite formulations.
\end{abstract}

\section{Introduction}

Nano-reinforcements are widely used to develop polymer nanocomposites (PNC) having better Thermo-mechanical and electromagnetic characteristics relative to the virgin host matrixes [1,2]. Some of the prominent features of nano-reinforcements are their high specific surface area, greater strength to weight ratio, and nano-level contact with the host polymeric chains [3]. To improve the required set of properties, nano-reinforcements are used in much lower quantity in the virgin polymer compared to micro/macro reinforcements. Carbon nanotubes (CNTs) stands at leading position among the nano-reinforcements family due to their exceptional features that are 1 Tera 
Pascal (elastic modulus) and 200 Giga Pascal (ultimate tensile strength) $10^{4}$ (aspect ratio), $1315 \mathrm{~m}^{2} / \mathrm{g}$ (specific surface area) and $1.3 \mathrm{~g} / \mathrm{cm}^{3}$ (density). Keeping in view of tremendous characteristics of CNTs, global market demand of this remarkable filler is enhanced day by day. Multiple polymer/ceramic/metal matrix nanocomposites filled with CNTs are used in variety of industries from sports to aerospace technology.

Composites incorporated with CNTs show good thermal oxidation resistance in air and nitrogen atmospheres, synergistically. Aspect ratio has great importance to introduce marvelous features into CNTs. Entanglement and agglomeration are the key problems in the way of nanotubes dispersion within the virgin polymer matrices. Variant CNTs activation and de-agglomeration methodologies have been developed for homogenously CNTs distribution throughout the matrix. Best methodology for CNTs treatment is considered for which aspect ratio would like to suffer minimum. Silane functionalization using appropriate silane coupling agent is one of the safer route to modify CNTs without aspect ratio sacrifice. [4]

Acrylonitrile butadiene rubber (NBR) has good thermal resistance, high tensile strength, good oil repellent, better hot air ageing and swelling resistance comparatively due to which it become an excellent candidate to fabricate automobile and oil industries composite parts. The prominent features of polydimethylsiloxane (PDMS) are good thermal endurance/flexibility, electrical/dielectric conductivity endorsed owing to the degree of polymerization and effect of crosslinking factor [5]. Silicone rubber has a large variety of applications from high temperature sealing to sensor technologies. It's thermal stability broad range $\left(-100\right.$ to $\left.310^{\circ} \mathrm{C}\right)$ makes it a suitable candidate for window/oven seals as well as in very low temperature environments such as on space vehicles. It has unlimited life cycle at moderate temperature conditions. Excellent mechanical performance with good resilience also adds beauty to its remarkable characteristics [6]. Some of the tremendous features of Styrene butadiene rubber (SBR) are good mechanical, abrasion, resilience, interfacial bonding with metals, tear/water/acid and flexibility at low temperatures. It's major area of applications are tyre, shoe soles and conveyer belt industries [7]. To enhance the thermomechanical, abrasion, adhesion, dielectric, electric, etc. characteristics, various types of reinforcements (silica, carbon, MWCNTs, alumina, clays, calcium/magnesium carbonate, etc.) are impregnated in the elastomeric matrix.

The present novel investigation reports the impact of $1 \mathrm{wt} \%$ silane modified MWCNTs on the thermal transmission/decomposition/quenching/transitions temperatures with respective enthalpies (glass/crystallization/melting) and mechanical characteristics of three different polymeric matrices (NBR, SR, SBR).

\section{Experimental}

Materials. Carbon black, Sulphur (S), zinc oxide $(\mathrm{ZnO})$ and stearic acid were received from Merck, Germany. MBTS; Mercaptobenzothiazole Disulphide and TMTD; Tetramethylthiuram Disulfide were delivered by Dalian Richon Chemical Co. Ltd, China. Aromatic Oil, DOP; dioctylphatalate and petroleum lubricant were obtained from International Petrochemicals (Pvt) Ltd, Pakistan. MWCNTs (prepared through CVD method using Fe as catalyst, diameter 10-40nm with length 40$60 \mu \mathrm{m}$ along 2400:1 aspect ratio, and purity $>95 \%$ ) were purchased from Nano-port Co. Ltd and China. NBR having grade name KumhoKNB35L was received from ABF International Corporation limited, Korea. SBR having grade INDOPOL 1502 was delivered by Evergreen Global PTE, Singapore. PDMS was supplied by Wacker, Germany. APTMS;3-aminopropyl-trimethoxysilane as silane coupling agent and $\mathrm{CTAB}$; cetrimonium bromide as cationic surfactant were supplied by from Sigma Aldrich, Germany.

Surface Modification of P-MWCNTs. Nondestructive surface activation route is adopted to functionalized pristine MWCNTs viz. silane modification method coupled with cationic surface capping. In this methodology, ingredients ratio in the solution (deionized water: APTMS: CTAB: PMWCNTs) is 90: 5: 3: 2 by weight percent. The solution is solicited at $40 \mathrm{KHz}$ and $85^{\circ} \mathrm{C}$ for $12 \mathrm{hrs}$. Then the nanotubes were filtered, washed, dried, and recovered to confirm functionalization and to incorporate into the elastomeric matrices. 
Fabrication of Rubber Nanocomposites. Nano-carbon and $1 \mathrm{wt} \%$ of F-MWCNTs were reinforced in the polymeric matrices (NBR, SBR, SR) using conventional rubber mixing techniques viz. internal dispersion kneader (temperature $\rightarrow 110^{\circ} \mathrm{C}$, router speed $\rightarrow 250 \mathrm{rpm}$, and time $\rightarrow 30 \mathrm{~min}$ ). The filled rubber amalgam was put on the two roller mixing mill (temperature $\rightarrow 70^{\circ} \mathrm{C}$, router speed $\rightarrow$ 40rpm, and time $\rightarrow 30 \mathrm{~min}$ ) to incorporate sulfur as a crosslinker, MBTS, HBS \& TMTD as accelerator, activators viz. ZnO \& stearic acid and plasticizers viz. aromatic oil, DOP \& wax in the elastomeric matrices viz. NBR and SBR [8]. For silicone rubber, dicumyl per oxide is used as a

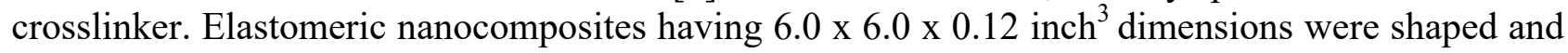
cured on hot isostatic hydraulic press (Pressure $\rightarrow 1400 \mathrm{psi}$, temperature $\rightarrow 140^{\circ} \mathrm{C}$ and time $\rightarrow 25 \mathrm{~min}$ ). Figure 1 demonstrates the formulation scheme of fabricated nanocomposites.

Characterization. FT-IR (Perkin-Elmer) was used to analyze the changes taken place on the nanotube surface due to the silane treatment in the frequency range $400-4000 \mathrm{~cm}^{-1}$. The degree of crystallinity of the Ps-MWCNTs and F-MWCNTs were characterized by powder X-ray diffraction (XRD). XRD measurements were carried out using an X-ray diffractometer (STOE, Germany) equipped with a back monochromator operating at $40 \mathrm{kV} \& 40 \mathrm{~mA}$ with copper cathode $(\lambda=0.154 \mathrm{~nm} \& \mathrm{a}=1.54060 \dot{\mathrm{A}})$ and scan angle from 10 to $80^{\circ}(2 \theta)$ with a scanning step of $0.02^{\circ} / \mathrm{s}$. Scanning electron microscopy (SEM, JSM 6940A, Jeol, Japan) along with the energy dispersive spectroscopy was used to observe the effect of silane modification, distribution of MWCNTs in the host polymer matrix, elemental analysis of the nanotubes and composites. Perkin-Elmer TG/DTA machine was run (Temperature range $\rightarrow 70$ to $900^{\circ} \mathrm{C}$, ramp rate $\rightarrow 10^{\circ} \mathrm{C} / \mathrm{min}$ and heating environment $\rightarrow$ oxygen) to elucidate the impact of functionalized nanotubes on the thermal decomposition behavior of the prepared composites. Perkin Elmer Diamond DSC, Japan was operated (heating rate $\rightarrow 10^{\circ} \mathrm{C} /$ minute, temperature span $\rightarrow-165$ to $450^{\circ} \mathrm{C}$, and heating atmosphere $\rightarrow$ air) to observe the thermal transitions $\left(\mathrm{T}_{\mathrm{g}}, \mathrm{T}_{\mathrm{c}}\right.$, and $\left.\mathrm{T}_{\mathrm{m}}\right)$ and also their relative specific enthalpies. Thermal conductivity $\left(\lambda_{N}\right)$ of the prepared specimens was evaluated on a domestically fabricated TC unit according to ASTM E1225-99 guidelines. Mechanical parameters viz. ultimate tensile strength, plasticity, and elastic modulus were conducted on UTM (AG-20KNXD Plus, Shimadzu) following ASTM D412-98A. Elastomeric hardness of FMWCNT/Polymer specimens were accomplished by using Torsee, Tokyo hardness testing machine.

\section{Results \& Discussion}

Surface Modification Analysis of MWCNTs. Fig. 2a shows the FTIR spectra of P-MWCNTs and F-MWCNTs. IR peaks at 1463.32, 2900, \& 1031.35 confirms the Si-O-CH3 moiety presence on the surface of the nanotubes [9]. Surface modification of F-MWCNTs is also proved from (10 to $80^{\circ}$

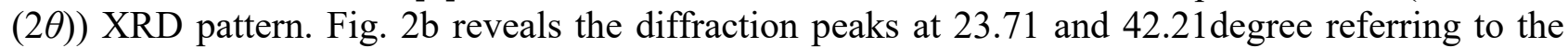
graphite structure derived from MWCNTs. Additionally, the intensity of (002) and (100) peaks of functionalized nanotubes are much closer to P-MWCNTs gives the confirmation for the fact that the surface treatment does not harm the graphene layer pattern. SEM/EDS analysis is showing surface morphology/composition of the P/F-MWCNTs. The proportional morphology analysis of P/FMWCNTs is carried out in Fig. 2(c) along with their elemental analysis confirming the oxidation of MWCNTs.

Dispersion of MWCNTs in Polymeric Matrices. Proper distribution of FMWCNTs in NBR, SBR, and SR matrices can be observed in the SEM micrographs (Fig. 1). Appropriate functionalization and high temperature mixing methodology have effectively enhanced the nanofiller diffusion/distribution/compatibility with the host polymer matrices. Two roller mixing mill technique that imparts longitudinal and transverse flow through the twin roll nip again and again, plays an important role to improve the nanotubes dispersibility within the elastomeric matrices. Good dispersion and compatibility of the active nanotubes make the fabricated nanocomposites more efficient regarding thermal and mechanical parameters. 


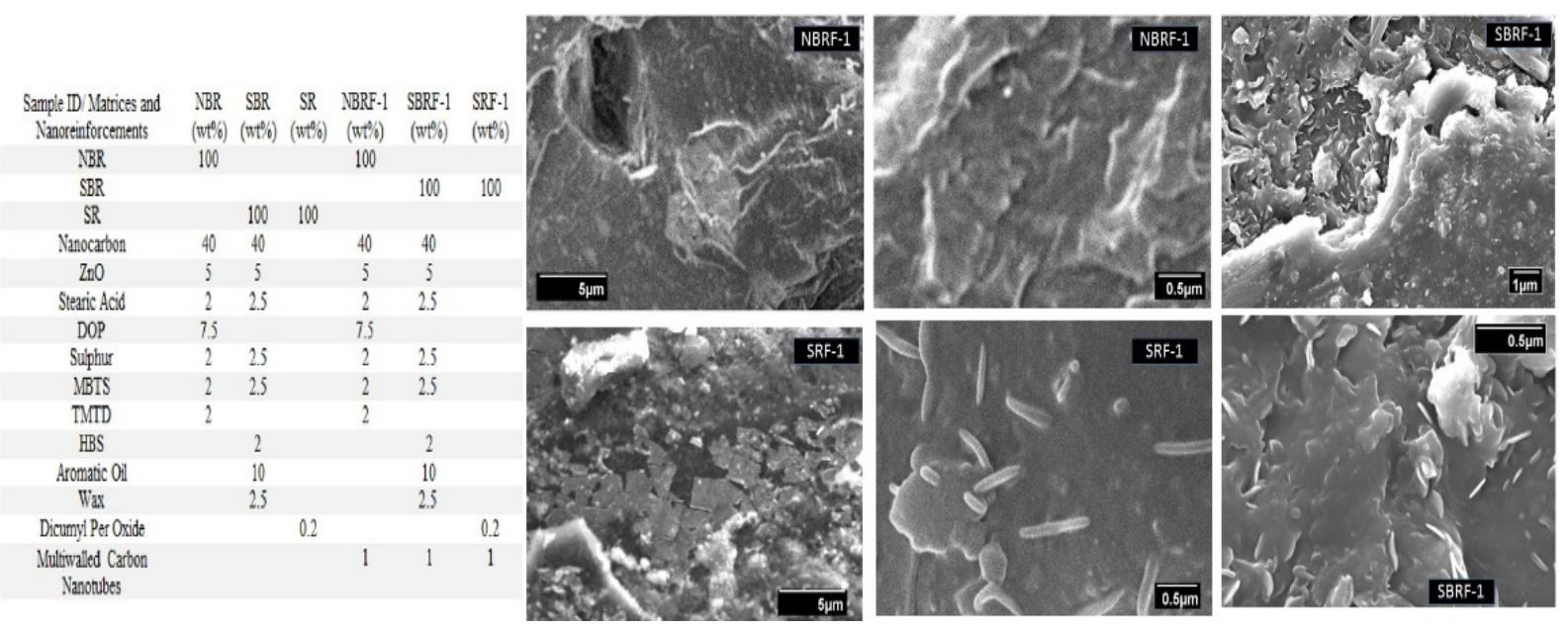

Fig. 1. Formulation of fabricated nanocomposite and distribution of F-MWCNTs within the polymer matrix at variant magnifications
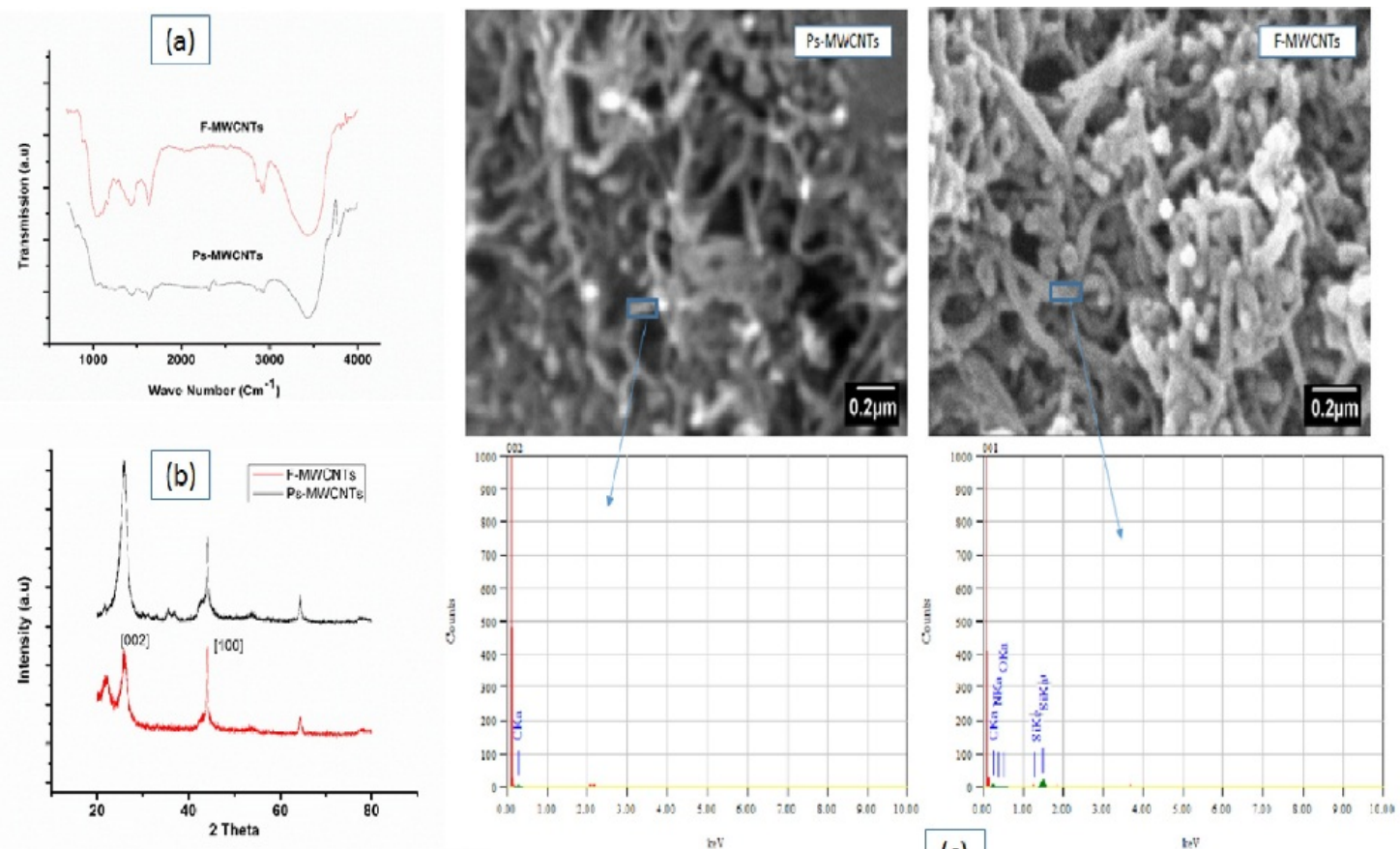

(c)

Fig. 2. FTIR spectra (a), XDR pattern (b) SEM micrographs along EDS analysis (c) for Ps-MWCNTs and F-MWCNTs

Thermal Properties. Fig. 3(a, b, c) demonstrates the thermal conductivity contours of the FMWCNTs doped polymer nanocomposites operated within a temperature span of 323523K.Although thermal conductivity of the incorporated nanotubes is much higher compared to the polymer matrices used in this study, but the experimental evaluation reports the reverse of it. It means that with increasing FMWCNTs concentration within the host polymer matrix, thermal conductivity value also increases correspondingly. How does it happen? Bryning et al. expressed that the thermal conductivities of the surfactant-SWNT/epoxy composites are much lesser (but the interfacial thermal resistance is higher) compared to that of the composites synthesized without surfactant at the same loading. With reference to the model proposed by Y. Agari that thermal conductivity of the polymeric composites (having high filler volume greater than $30 \mathrm{wt} \%$ ) is reduced with increasing temperature. The decrease is attributed due to the heat absorption through phonon entrapping/capturing by the impregnated nanotubes [10]. Even dispersion of the incorporated nanotubes also imparts an important role to dissipate the impact of incoming heat flux. Therefore, properly dispersed F-MWCNTs establish nanotubes-polymeric chains network that offers resistance to phonon transmission through the composites specimens and consequently reduces thermal 
conductivity as well[10]. Minimum value of thermal conductivity is scrutinized for F-MWCNT/SR formulation in comparison the other tested scheme. The presence of another carbonaceous filler viz. carbon black (40 wt\% in each system) synergistically communicates its role along with the nanotubes to minimize thermal transport through the fabricated specimens. The reason behind the maximum thermal resistance of the nanotubes doped silicon rubber composite is set behind its better thermal insulation/stability character compared the rest elastomeric systems [11].

Thermal oxidation of the polymer composites is an important parameter to validate the operating temperature range of the materials. Figure 3(d, e, f) illustrates the thermal decomposition curves of the fabricated specimens (NBR loaded with FMWCNTs and without the nanotubes) within the temperature range of 25 to $800^{\circ} \mathrm{C}$. The very first thermal decomposition step is observed from 200 to $400^{\circ} \mathrm{C}$ that may be attributed due to the exhaust of the incorporated aromatic oil, processing aids viz. stearic acid and wax. Further temperature increment of $100^{\circ} \mathrm{C}$ causes major polymer degradation due to polymeric chain scission. FMWCNTs incorporation improves thermal stability level of NBR up to $5 \%$ due to proper dispersion and compatibility of the nanotubes with the host matrix[12]. Figure $6 \mathrm{~b}$ shows the thermal decomposition behavior of SBR composites (one have FMWCNTs and other have not). Major decomposition zone in this study is observed within the temperature premises viz. $400-600^{\circ} \mathrm{C}$ due to host matrix charring. Nanotubes loading effective enhance the thermal endurance level up to $7 \%$ attributed to properly developed FMWCNTs-SBR network that offers resistance to the incoming heat flux [13]. Fig. 3f elucidates maximum reinforcing effect of FMWCNTs regarding thermal stability improvement (40\%) relative to the virgin silicon rubber matrix. As the pristine MWCNTs are functionalized using APTMS which introduces silane moiety on the surface of the nanotubes, that has higher chemical affinity and compatibility with the SR matrix relative the rest matrices. Consequently, it's impact to improve the thermal stability is observed much higher for SR in comparison with NBR and SBR systems.

DSC study in Fig. $3(\mathrm{~g}, \mathrm{~h}, \mathrm{i})$ demonstrates impact of FMWCNTs on thermal transition temperatures $\left(\mathrm{T}_{\mathrm{g}}, \mathrm{T}_{\mathrm{c}}\right.$, and $\mathrm{T}_{\mathrm{m}}$ ) of the elastomeric composites. The accumulated data (using DSC curves) of these phase transformation are collected in Table 1. Glass transition temperature viz. $T_{g}$ is diminished with the impregnation of FMWCNTs in the host elastomeric matrix. It is attributed due to the CNT's network in the elastomeric matrices that offers resistance to the phase transformations by interacting with the host elastomeric chains at the molecular scale and widens the plastic range of the elastomeric composites. It indicates that the nanotubes incorporated elastomeric composites can be regulated in a more negative temperature road map I comparison with the composites without nanotubes loading. The improvement in the crystallization $\left(\mathrm{T}_{\mathrm{c}}\right)$, and melting temperatures $\left(\mathrm{T}_{\mathrm{m}}\right)$ of the elastomeric composites is due to resistance offered by the nanotubes that slower down the thermal chain mobility by entrapping phonons within the polymer-FMWCNTs network. Specific enthalpies of the elastomeric composites are portrayed in Table 1[11].

Glass transition/crystallization transformations are exothermic phenomena but melting of the elastomeric composite is an endothermic phenomenon. It is noticed that specific enthalpies of the glass transition phase changes are reduced with increasing nanotubes to matrix ratio. But the specific enthalpies of crystallization, onset and peak melting phases are enhanced due to the enhancement in endothermic nature of the composites with increasing the nanotubes doping concentration in the host elastomeric matrix.

Table 1. Glass transition, crystallization, first melting phase and second melting phase temperatures and their respective specific enthalpies of all fabricated nanocomposites

\begin{tabular}{|c|c|c|c|c|c|c|}
\hline Sample ID & NBR & NBRF-12 & SBR & SBRF-1 & SR & SRF-1 \\
\hline Glass transition Temperature $T_{g} /{ }^{\circ} \mathrm{C}$ & $-11.5 \pm 0.1$ & $-18.8 \pm 0.1$ & $-36.6 \pm 0.1$ & $-42.9 \pm 0.1$ & $-131.1 \pm 0.1$ & $-144.7 \pm 0.1$ \\
\hline Crystallization Temperature $T_{c} /{ }^{\circ} \mathrm{C}$ & $97.95 \pm 0.1$ & $91.62 \pm 0.1$ & $86.98 \pm 0.1$ & $82.07 \pm 0.1$ & $-92.71 \pm 0.1$ & $-87.41 \pm 0.1$ \\
\hline Melting Temperature $T_{m l} /{ }^{\circ} \mathrm{C}$ & $179.69 \pm 0.15$ & $191.82 \pm 0.25$ & $206.55 \pm 0.1$ & $208.11 \pm 0.1$ & $-76.286 \pm 0.1$ & $-70.763 \pm 0.1$ \\
\hline Melting Temperature $T_{m 2} /{ }^{\circ} \mathrm{C}$ & $366.12 \pm 0.1$ & $368.44 \pm 0.1$ & $371.47 \pm 0.1$ & $372.77 \pm 0.1$ & $-44.87 \pm 0.1$ & $-43.48 \pm 0.1$ \\
\hline Specific Enthalpy of Glass transition $/ \mathrm{Jg}^{-1}$ & $6.18 \pm 0.01$ & $4.39 \pm 0.01$ & $3.51 \pm 0.01$ & $2.58 \pm 0.01$ & $0.81 \pm 0.01$ & $0.22 \pm 0.01$ \\
\hline Specific Enthalpy of Crystallization $/ \mathrm{Jg}^{-1}$ & $6.46 \pm 0.01$ & $7.04 \pm 0.01$ & $14.13 \pm 0.01$ & $16.52 \pm 0.01$ & $7.42 \pm 0.01$ & $8.75 \pm 0.01$ \\
\hline Specific Enthalpy of Onset Melting Phase $/ \mathrm{Jg}^{-1}$ & $7.46 \pm 0.01$ & $8.59 \pm 0.01$ & $8.36 \pm 0.01$ & $10.92 \pm 0.01$ & $7.12 \pm 0.01$ & $19.69 \pm 0.01$ \\
\hline Specific Enthalpy of Peak Melting Phase $/ \mathrm{Jg}^{-1}$ & $31436 \pm 0.1$ & $375.26 \pm 0.1$ & $174.29 \pm 0.01$ & $180.65 \pm 0.01$ & $2.46 \pm 0.01$ & $7.92 \pm 0.01$ \\
\hline
\end{tabular}



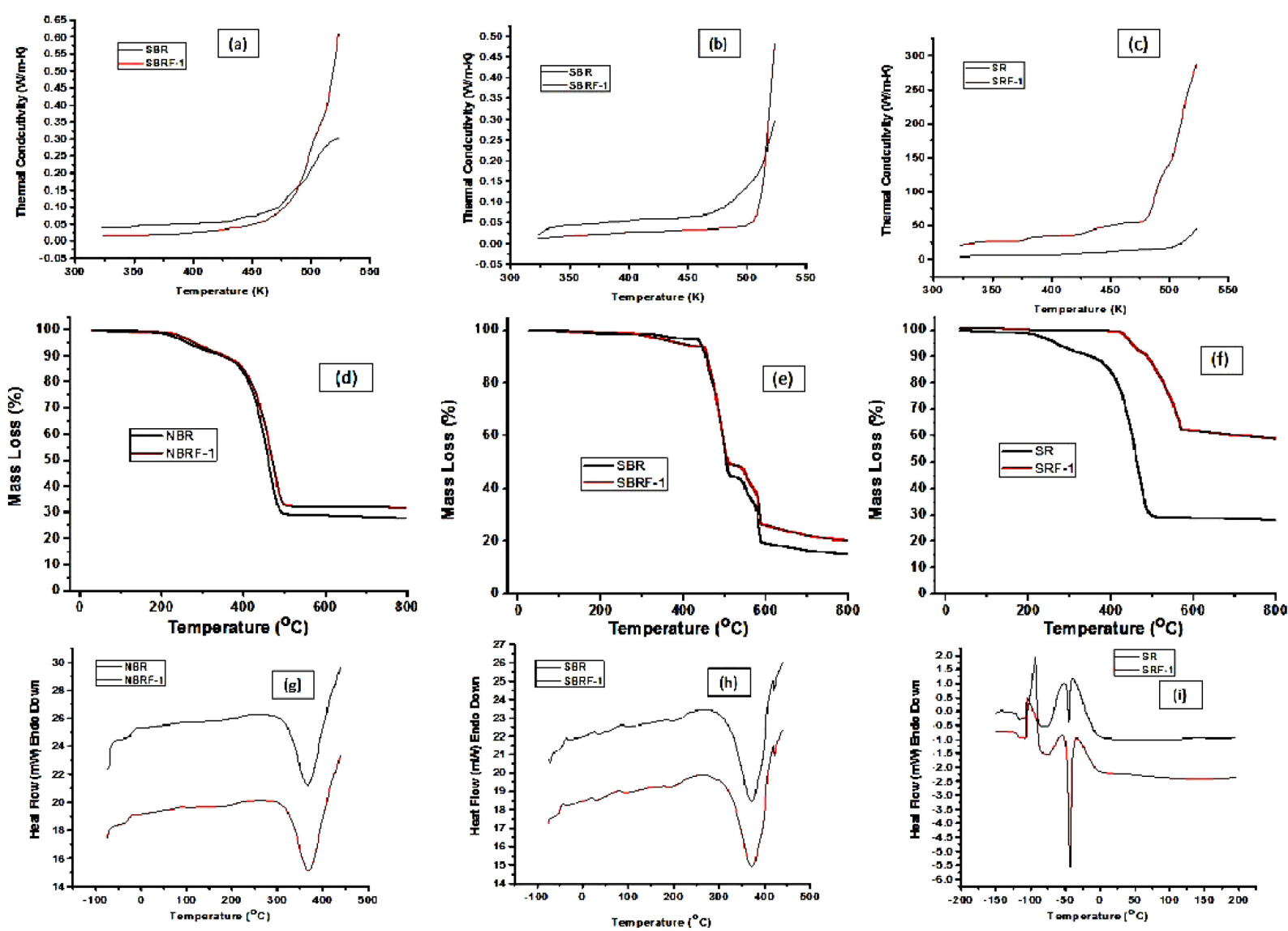

Fig. 3. Thermal decomposition analysis (a,b,c), Thermal conductivity behavior (d,e,f,), DSC contours $(\mathrm{g}, \mathrm{h}, \mathrm{i})$ of the fabricated nanocomposites

Mechanical Properties. Stress-strain curves of the elastomeric composites are depicted in Fig. 4 that demonstrates an improvement in mechanical strength while elongation at break reduces with the incorporation of FMWCNTs in the host elastomeric matrices. Bokobza et al. measured the tensile strength 5.5 MPa. Perez et al. [14] prepared MWCNT/NBR nanocomposites with improved tensile strength (UTS is $36 \%$ higher). Girun et al. reported UTS around 1.25MPa. Table 4 shows that $1 \mathrm{wt} \%$ impregnation of F-MWCNTs in the NBR matrix elevates the tensile strength and elastic modulus up to 30 and $1.18 \%$, respectively. It is attributed to proper dispersion of the nanotubes in the host elastomeric matrix, secondly covalent interaction between the F-MWCNT/polymer, and third factor is the exceptional mechanical strength of the CNTs. Figure 4 simulates the effect of FMWCNTs on the elastomeric hardness of the prepared nanocomposites. Polymeric hardness is improved according to the descending order SBRF-1<NBRF-1<SRF-1. The addition of FMWCNTs in the host polymeric matrices enhances the rubber hardness due to strong filler to matrix interaction/compatibility attributed to the presences of silane functional group presence on the surface of the nanotubes. Another reason of hardness improvement is the decrease of the polymeric chain mobility with the addition of F-MWCNTs in the elastomeric matrices as additional crosslinking and bridging is introduced in the composite structure due to the presence of functional nanotubes bridge among the host polymer matrix chain $[8,15,16]$. 


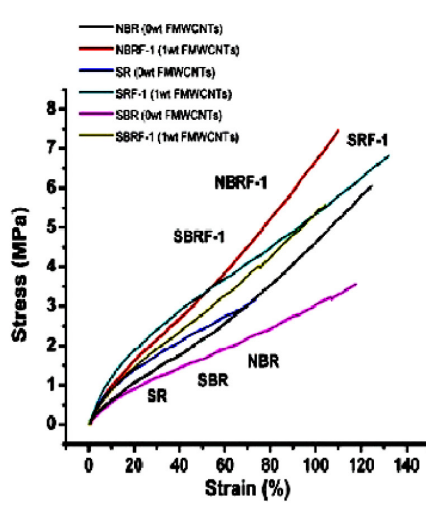

$\begin{array}{cccc}\begin{array}{c}\text { Sample ID/ } \\ \text { Mechanical } \\ \text { Properties }\end{array} & \begin{array}{c}\text { Ultimate } \\ \text { Tensile Strength } \\ (\mathrm{MPa})\end{array} & \begin{array}{c}\text { Elongation at } \\ \text { Break (\%) }\end{array} & \begin{array}{c}\text { Hardness } \\ \text { (Shore A) }\end{array} \\ \text { NBR } & 5.172 \pm 0.01 & 108.605 \pm 0.01 & 51.5 \pm 0.01 \\ \text { NBRF-1 } & 7.442 \pm 0.01 & 110.861 \pm 0.01 & 61.4 \pm 0.01 \\ \text { SBR } & 3.324 \pm 0.01 & 102.017 \pm 0.01 & 70.2 \pm 0.01 \\ \text { SBRF-1 } & 5.527 \pm 0.01 & 103.437 \pm 0.01 & 76.3 \pm 0.01 \\ \text { SR } & 3.178 \pm 0.01 & 73.652 \pm 0.01 & 50.6 \pm 0.01 \\ \text { SRF-1 } & 5.474 \pm 0.01 & 101.157 \pm 0.01 & 60.5 \pm 0.01\end{array}$

Fig. 4. Effect of different doping concentrations of F-MWCNT on stress-strain performance of fabricated nanocomposites

\section{Conclusions}

- IR transmission spectrum confirms the $\mathrm{Si}-\mathrm{O}-\mathrm{CH}_{3}$ attachment on the surface of the nanotubes confirming the adopted methodology is a good approach to functionalize the pristine MWCNTs.

- Molecular structural study of the treated/untreated MWCNTs illustrates the graphitic structure confirmation and preservation even after the functional group attachment with the nanotubes.

- Microscopic study reveals the proper dispersion of F-MWCNTs in the host elastomeric matrices that has key importance to improve the thermo-mechanical characteristics of the prepared nanocomposites. Compositional analysis of the P/F-MWCNTs demonstrates the successful addition of oxygen and silicone due to the presence of silane functional group on the surface of the carbon nanotubes.

- Experimentally, determine thermal conductivity values shows that the addition of FMWCNTs is effectively reduced thermal transmission through the nanocomposites attributed due to the phonon entrapment and high carbonaceous filler loading in the host elastomeric matrices.

- Comparative thermal oxidation analysis indicates thermal endurance enhancement due to the F-MWCNTs incorporation in the host elastomeric matrices and the maximum thermal stability improvement is observed for nanotube doped silicone rubber nanocomposites due to better filler to matrix compatibility in comparison with the rest formulations.

- Thermal phase transition study describes reduction in glass transition temperature while crystallization and melting temperature promote to higher values due to the nanotube $\mathrm{s}$ impregnation in the host matrices.

- The addition of F-MWCNTs has remarkably improved elastic modulus of the elastomeric nanocomposites while plasticity suffers in this practice.

- Well dispersed F-MWCNTs effectively enhanced Shore A rubber hardness of the polymer nanocomposites attributed due to the decrease in polymeric chain mobility which in interlink with the increment in crosslinking density

\section{Acknowledgement}

The authors would like to greatly acknowledge Research Grant Fiscal Year (2017-2018), University of the Punjab, Lahore, Pakistan for providing sufficient funds to execute this research. 


\section{References}

[1] S. Sagar, N. Iqbal, A. Maqsood, M. Bassyouni, MWCNTS incorporated natural rubber composites: thermal insulation, phase transition and mechanical properties, Int. J. Eng. Technol., 6 (2014) 168-173.

[2] S. S. Iqbal, F. Inam, N. Iqbal, T. Jamil, A. Bashir, M. Shahid, Thermogravimetric, differential scanning calorimetric, and experimental thermal transport study of functionalized nanokaolinitedoped elastomeric nanocomposites, J. Therm. Anal. Calorim., 125 (2016) 871-880.

[3] M. A. Mansilla, L. Silva, W. Salgueiro, A. J. Marzocca, A. Somoza, A study about the structure of vulcanized natural rubber/styrene butadiene rubber blends and the glass transition behavior, J Appl Polym Sci., 125 (2012) 992-999.

[4] S. S. Iqbal, N. Iqbal, T. Jamil, A. Bashir, Z. M. Khan, Tailoring in thermomechanical properties of ethylene propylene diene monomer elastomer with silane functionalized multiwalled carbon nanotubes, J Appl Polym Sci., (2015).

[5] D. R. Paul, J. E. Mark, Fillers for polysiloxane (silicone) elastomers, Prog. Polym. Sci., 35 (2010) 893-901.

[6] N. Iqbal, M.B. Khan, S. Sagar, A. Maqsood, Fabrication and characterization of multiwalled carbon nanotubes/silicone rubber composites, J. Appl. Polym. Sci., (2012).

[7] K. A. Dubey, Y. K. Bhardwaj, C. V. Chaudhari, N. K. Goel, S. Sabharwal, K. Rajkumar, S.K. Chakraborty, Radiation effects on styrene-butadiene-ethylene-propylene diene monomer-multiple walled carbon nanotube nanocomposites, Polym. Adv. Technol., 22 (2011) 1888-1897.

[8] N. Iqbal, M. B. Khan, S. Sagar, A. Maqsood, Fabrication and characterization of multiwalled carbon nanotubes/silicone rubber composites, J. Appl. Polym. Sci., 128 (May 2013) 2439-2446.

[9] M. V. Naseh, A. Khodadadi, Y. Mortazavi, O. A. Sahraei, F. Pourfayaz, S. M. Sedghi, Functionalization of carbon nanotubes using nitric acid oxidation and DBD plasma, World Academy of Sci. Eng. Technol., 49 (2009) 177-179.

[10] A. E. Aliev, M. H. Lima, E. M. Silverman, R. H. Baughman, Thermal conductivity of multiwalled carbon nanotube sheets: radiation losses and quenching of phonon modes, Nanotechnol., 21 (2010) 035709.

[11] S. Sagar, N. Iqbal, A. Maqsood, U. Javaid, Thermogravimetric, differential scanning calorimetric and experimental thermal transport study of MWCNT/NBR nanocomposites, J. Therm. Anal. Calorim., 114 (2013) 161-167.

[12] S. Sagar, N. Iqbal, A. Maqsood, Multiwalled carbon nanotubes impregnated rubber nanocomposites: thermal transport/decomposition and differential scanning calorimetric study, J. Reinfor. Plast. Comp., 32 (2013) 1052-1061.

[13] Y. T. Wang, C. S. Wang, H. Y. Yin, L. L. Wang, H. F. Xie, R. S. Cheng, Carboxyl-terminated butadiene-acrylonitrile-toughened epoxy/carboxyl-modified carbon nanotube nanocomposites: Thermal and mechanical properties, Express Polym. Lett., 6 (2012) 719-728.

[14] L. D. Perez, M. A. Zuluaga, T. Kyu, J. E. Mark, B. L. Lopez, Preparation, Characterization, and Physical Properties of Multiwall Carbon Nanotube/Elastomer Composites, Polym. Eng. Sci., 49 (May 2009) 866-874.

[15] N. Iqbal, S. Sagar, M. B. Khan, M. I. Bassyouni, Z. M. Khan, Aluminum silicate fibers impregnated acrylonitrile butadiene rubber composites: Ablation, thermal transport/stability, and mechanical inspection, J. Appl. Polym. Sci., 130 (2013) 4392-4400.

[16] S. Sagar, N. Iqbal, A. Maqsood, M. Shahid, N.A. Shah, T. Jamil, M.I. Bassyouni, Fabrication and thermal characteristics of functionalized carbon nanotubes impregnated polydimethylsiloxane nanocomposites, J. Comp. Mater., (2014) DOI:10.1177/ 0021998314528733. 